harnstoff für die Ansicht, dafs sic kein wahrer Rhodankörper mehr, sondern eine Verbindung ist, wo Kohlenstoff und Schwefel in Form von (CS) fungiren, wie folgende Formel symbolisch ausdrūckt :

$$
\left[\begin{array}{c}
(\mathrm{CS})^{\prime \prime}(\mathrm{NH})^{\prime \prime} \\
(\mathrm{NH})^{\prime \prime}(\mathrm{CS})^{\prime \prime}
\end{array}\right]^{\prime \prime \prime}
$$

Die zwei Sulfocarhoxyle und die beiden Imide sind in der Art zusammengelagert, dafs ihrem vereinigten Molecul noch zwei freie Affinitäten übrig bleiben, welche den zweiwerthigen Schweful sättigen. Die beim Bildungsprocefs des Schwefelharnstoffs bewirkte Abspaltung von Schwefelkohlenstoff ist dadurch gut erklärt und durch die beiden Imidwasserstoffe wird die Bibasicität der Sāure leicht begreiflich.

Leipzig, im August 1869.

\title{
Aus dem chemischen Laboratorium des Prof. Strecker.
}

Ueber einige Sulfosäuren des Benzyls; von Dr. Otto Bühler.

\section{Benzylsulfosüure.}

Im Benzol und seinen Homologen lassen sich durch Behandlung mit rauchender Schwefelsāure ein oder mehrere Wasserstoffatome durch die Gruppe $\mathrm{SO}_{3} \mathrm{H}$ vertreten, wodurch Sulfosäuren der aromatischen Kohlenwasserstoffe entstehen. Diese Substitution findet stets im Kerne des Kohlenwasserstoffes Statt. Es war bis jetzt aber keine Methode bekannt, die Wasserstoffatome der Seitenkette durch $\mathrm{SO}_{3} \mathrm{H}$ zu ersetzen. 
Herr Prof. Strecker hat in Jahre 1865 eine wichtige allgemeine Darstellungsweise der Sulfosäuren entdeckt, nämlich durch Einwirkung neutraler schwefligsaurer Alkalien auf die Chloride, Bromide oder Jodide der Kohlenwasserstoffe. Auf seine Veranlassung hin habe ich versucht, Sulfosäuren aus dem Toluol darzustellen, worin in der Seitenkette Wasserstoff durch $\mathrm{SO}_{3} \mathrm{H}$ vertreten ist. $\mathrm{Zu}$ diesem Zweck liefs ich nach seiner oben angegebenen Methode auf Benzylchlorid, Chlorbenzylchlorid und Chlorobenzol neutrales schwefligsaures Kali einwirken, und theile in Folgendem die hierbei gewonnenen Resultate mit.

Zur Darstellung der oben erwähnten gechlorten Producte wurde reines Toluol in eine aufwärts gerichtete, mit einem Kühlapparat verbundene Retorte gebracht, zum Sieden erhitzt und durch den Tubulus $z u$ den Toluoldãmpfen ein Strom von Chlorgas geleitel. Nach etwa 6 stündigem Kochen wurde die Operation unterbrochen und das Product rectificirt. Anfangs destillirte Toluol über, welches nochmals mit Chlor behandelt wurde. Das hierauf bei $175^{\circ} \mathrm{C}$. übergehende Benzylchlorid wurde getrennt von dem bei $200^{\circ}$ siedenden Chlorbenzylchlorid aufgefangen. Gleichzeitig mit diesen Körpern traten auch Monochlortoluol und höher gechlorte Verbindungen des Toluols auf.

Benzylsulfosaures Kali. - Das auf diese Weise erhaltene Benzylchlorid $\mathrm{C}_{6} \mathrm{H}_{5} \cdot \mathrm{CH}_{2} \mathrm{Cl}$ brachte ich mit einer äquivalenten Menge neutralen schwefligsauren Kali's in ziemlich concentrirter Lösung in eine aufwärts gerichtete Retorte, verband den Hals derselben mit einem ebenfalls aufwärts gerichteten Kühlapparat und erhitzte zum Sieden. Nach mehrstündigcm Kochen war die ölartige, oben schwimmende Schicht von Benzylchlorid größstentheils verschwunden und wurde daher die Einwirkung unterbrochen. 
Die etwas trübe Flüssigkeit wurde filtrirt und auf dem Wasserbade eingeengt. Beim Erkalten ersiarrte sie zu einem Brei von farblosen glänzenden Krystallschuppen, welche durch mehrmaliges Umkrystallisiren von beigemengtem Chlorkalium befreit wurden. Das reine Salz bildet farblose, gut ausgebildete gerade rhombische Säulen.

1,3450 Grm. Substanz, auf 100 bis $150^{\circ}$ crhitzt, verloren 0,1175 Wasser, was $8,73 \mathrm{pC}$. entspricht.

$0,4071 \mathrm{Grm}$. getrocknete Substanz wurden mit concentrirter Scluwefolsüure behandelt, geglüht und gaben 0,1647 schwefelsaures Kali.

0,3470 Grm. getrocknete Substanz gaben nach der Verbromnung mit chromsaurem Blei 0,5130 Kohlensaure und 0,1125 Wasser.

0,4248 Grm. getrocknete Substanz wurden zar Schwefelbestimmung mit Kalihydrat und Salpeter geschmolzen, in Wasser gelöst, mit Salzsăuro angesăuert, mit Chlorluaryum gefailt und gaben 0,4715 schwefelsauren Baryt.

Aus vorstehenden Resultaten leitet sich die Formel $\mathrm{C}_{7} \mathrm{H}_{7} \mathrm{SO}_{3} \mathrm{~K}$ ab, wie folgende Zusammenstellung der berechneten und gefundenen procentischen Werthe zeigt :

\begin{tabular}{lrrc} 
& \multicolumn{2}{c}{ Berechnet } & Gefunden \\
\cline { 2 - 4 } $\mathrm{C}_{7}$ & 84 & 40,00 & 40,31 \\
$\mathrm{H}_{7}$ & 7 & $\mathbf{3 , 3 3}$ & 3,60 \\
$\mathrm{~S}$ & $\mathbf{3 2}$ & 15,24 & 15,25 \\
$\mathrm{O}_{8}$ & 48 & 22,86 & - \\
$\mathrm{K}$ & $\mathbf{3 9}$ & 18,57 & 18,13 \\
\cline { 2 - 3 } & $\mathbf{2 1 0}$ & $100,00$. &
\end{tabular}

Aufserdem enthält das Salz noch 8,7 pC. Krystallwasser, was einem Molecule entspricht. Berechnet 7,9 pC.

Benzylsulfosaurer Baryt. - Durch doppelten Austausch stellte ich aus dem Kalisalz das Barytsalz dar, indem ich die Lōsung des Kalisalzes mit einer berechneten Menge Chlorbaryum versetzle. Das reine Barytsalz bildet farblose blätterige Krystalle, die sich ziemlich schwer in Wasser lösen.

Die Analyse derselben ergab Folgendes : 
0,6530 Grm. Substanz verloren, auf 100 bis $150^{\circ}$ erhitzt, 0,0462 Wasser, was einem Procentgehalt von 7,08 entspricht.

Zur Bargumbestimmung wurden 0,3558 Grm. getrocknete Substanz mit concentrirter Schwefelsäure übergossen und geglüht. Sie gaben 0,1745 schwefelsauren Baryt, woraus sich ein Baryumgchalt von $28,83 \mathrm{pC}$. berechnet. Nach der Formel $\mathrm{C}_{7} \mathrm{H}_{7} \mathrm{SO}_{3} \mathrm{Ba}^{*}$ ) whiro dersclbe $28,60 \mathrm{pC}$.

Der Wassergehalt, der 7,08 pC. betrug, entspricht wie im Kalisalze einem Molecule. Berechnet 7,00 pC.

Basiscll-benzylsulfosaures Blei. - Das Barytsalz verwandte ich als Ausgangspunkt zur Gewinnung verschiedener Salze.

Zur Darstellung des Bleisalzes wurde mit Schwefelsäure der Baryt aus der Salzlösung ausgefällt und das Filtrat so lange mit Bleioxydhydrat digerirt, bis sich dasselbe nicht mehr auflöste.

Beim Erkalten entstand ein stark glänzender krystallinischer Niederschlag, der sich durch folgende Analyse als ein basisches Bleisalz ergab :

$0,7070 \mathrm{Grm}$. Substanz verloren, auf 100 bis $130^{\circ}$ erhitzt, 0,0180 Wasser, entsprechend $2,55 \mathrm{pC}$.

Zur Bleibestimmung wurdon $0,6890 \mathrm{Grm}$. getrocknete Substanz in Wasser gelöst, mit cinem Ueberschufs von Schwefelstiure und dann mit Weingeist übergossen. Nach dem Trocknen und Wägen gaben sie 0,5315 schwcfelsaures Blei, entsprechend 52,70 pC. $\mathrm{Pb}$.

Aus der Formel $\mathrm{C}_{6} \mathrm{H}_{5} \cdot \mathrm{CH}_{2} \cdot \mathrm{SO}_{2} \mathrm{~Pb}+\mathrm{PbHO}$ berechnet sich der Bleigehalt auf 52,41 pC. Der Wassergehalt betrug 2,55 pC., was $1 / 2$ Molecul entspricht. Berechnet 2,23 pC.

Aus diesem Bleisalz liefs sich durch Kohlensãure die Hälfte des Blei's entfernen, wodurch ein neutrales benzylsulfosaures Bleisalz in Gestalt von glānzenden wasserfreien Krystallblättern erhalten wurde.

*) In dieser und den folgenden Formeln bezeichnen die Symbole der Metalle ihre Aequivalente und nicht ihre Atorngewichte. 
0,8045 Grm. Substanz nahmen beim Erhitzen auf $150^{\circ}$ nicht ab und wurden daher zur Bleibestimmung verwandt. Sie galsen 0,4435 schwefcls. Blei, was $37,66 \mathrm{pC}$. Blei entspricht. Berechnet aus der Formol $\mathrm{C}_{6} \mathrm{H}_{5} \cdot \mathrm{CH}_{2} \cdot \mathrm{SO}_{8} \mathrm{~Pb} 37,70 \mathrm{pC}$. Blei.

Benzylsulfosaures Ammoniak- und Kallssalz. - Ersteres wurde erhalten, indem ich die Lösung des Barytsalzes so lange mit kohlensaurem Ammoniak versetzte, bis sämmtlicher Baryt ausgefällt war. Beim Eindampfen des Filtrates schied sich das Ammoniaksalz in farblosen, sehr leicht löslichen Krystallblättern aus.

Ich verwandte dasselbe zur Darstellung des Kalksalzes. Zu diesem Zweck wurde die Ammoniaksalzlösung mit Kalkmilch gekocht, bis kein Ammoniak mehr entwich. Durch Einleiten von Kohlensäure wurde der überschüssig zugesetzte Kalk ausgefällt und das Filtrat auf dem Wasserbade eingedampft. Beim Erkalten schied sich das Kalksalz in farblosen, dem Barytsalz gleichenden Krystallblättern aus, welche folgende Zusammensetzung hatten :

0,9400 Grm. Substanz, auf 100 bis $150^{\circ}$ erhitzt, verloren 0,0810 Wasser, entsprochend $8,62 \mathrm{pC}$.

0,8590 Grm. getrocknete Substanz gahen nach der Behandlung mit Schwefelsïure im Platintiegel 0,3070 schwefelsauren Kalk, entsprechond $10,51 \mathrm{pC}$. Ca.

Aus der Formel $\mathrm{C}_{6} \mathrm{H}_{5}, \mathrm{CH}_{2} . \mathrm{SO}_{3} \mathrm{Ca}$ berechnet sich der Calciumgehult zu $10,47 \mathrm{pC}$.

Der Wassergehalt wurde gleich $8,62 \mathrm{pC}$. gefunden, was einem Molecule entspricht. Berechnet 8,57 pC.

Benzylsulfosäure. - Die freie Säure stellte ich aus dem Bleisalz dar, indem ich in die Lösung desselben so lange Schwefelwasserstoff einleitete, bis sich alles Blei als Schwefelblei abgeschieden hatte. Das Filtrat wurde auf dem Wasserbade eingedampft, wobei ein dicker Syrup hinterblieb. Nach mehrtägigem Stehen unter der Luftpumpe neben Schwefelsăure erstarrte derselbe zu farblosen Krystallen. Dieselben waren von äufserst hygroscopischer Beschaffenheit, weshalb 
ihre Analyse unterblieb. Auch war die Zusammensetzung der Säure nach den vielfachen Analysen ihrer Salze unzweifelhaft.

Benzylsulfosaures Silber. - Zur Darstellung desselben wurde die Lösung der freien Sãure so lange mit Silberoxyd erhitzt, bis sich dasselbe nicht mehr löste. Aus dem Filtrat schied sich beim Erkalten das Salz in farblosen wasserfreien Krystallschuppen aus, die sich am Licht leicht schwärzten.

0,5170 Grm. Substanz nahmen beim Erhitzen auf $100^{\circ}$ nicht ab. Sio gaben bei der Silberbestimmung 0,2612 Chlorsilber, entsprechend 38,01 pC. Ag.

Nach der Formel $\mathrm{C}_{8} \mathrm{H}_{5} \cdot \mathrm{CH}_{2} \cdot \mathrm{SO}_{3} \mathrm{Ag}_{6}$ berechnet sich der Silbergehalt auf $38,71 \mathrm{pC}$.

Auch das Kupfersalz versuchte ich darzustellen, indem ich die Lösung des Silbersalzes mit Kupferchlorid versetzte. Die voun Chlorsilber ahfiltrirte und eingeengte Lósung krystallisirte schwierig und war das erhaltene Salz auch nach oftmaligem Umkrystallisiren aus Alkohol nicht rein zu gewinnen.

\section{Nitrobenzylsulfosäure.}

Um die Benzylsulfosăure zu nitriren, wurde das Barytsalz derselben mit rauchender Salpetersäure behandelt. Es bildete sich eine gelbliche zusammengesinterte Masse von nitrobenzylsulfosaurem Baryt, der durch Umkrystallisiren aus heifsem Wasser in farblosen, glänzenden Nadeln erhalten wurde :

0,4192 Grm. Substanz verloren beim Erhitzen auf $160^{\circ} 0,0247$ Wasser, entsprechend $5,8 \mathrm{pC}$.

0,3945 Grm. getrocknete Substanz gaben bei der Bohandlung mit concentrirter Schwefelsh̆urc 0,1595 schwefelsauren Baryt, entsprechend 23,98 pC. Baryum.

\left. Aus der Formol ${\stackrel{\mathrm{CO}}{6} \mathrm{H}_{4}}_{\mathrm{NO}_{2}}^{\mathrm{p}_{2}}\right\} \mathrm{CH}_{2} \mathrm{SO}_{8} \mathrm{Ba}$ berechnen sich 24,08 pC. Baryam.

Der Wassergehalt, welcher $5,8 \mathrm{pC}$. betrug, entspricht einem Molecule; berechnet $5,9 \mathrm{pC}$. 
Basisches und neutrales Bleisalz. - Aus dem Barytsalz wurden zwei Bleisalze gewonnen, ein basisches und ein neutrales. Ersteres erhielt ich, indem aus der Salzlōsung der Baryt mit Schwefelsäure ausgefält und die entstandene freie Säure mit einem Ueberschuls von Bleioxydhydrat behandelt wurde. Nach dem Filtriren schieden sich farblose, in kaltem Wasser schwer lösliche Krystalle des basischen Salzes aus.

Die Wasser- und die Bleibestimmung gaben folgende Werthe.

0,9005 Grin. Substanz, erhitzt auf $120^{\circ}$, gaben 0,0365 Wasserverlust, was $4 \mathrm{pC}$. entspricht.

Zur Bleibestimmung wurden 0,3980 Grm. getrocknete Substanz im Porccllantiegol mit concentrirter Schwefelsture behandelt und gaben $0,2760 \mathrm{Grm}$. schwefelsatres Blei, entsprechend 47,36 pC. Blei. Bercelinet aus der Formel $\left.\underset{\mathrm{NO}_{2}}{\mathrm{C}_{6} \mathrm{H}_{4}}\right\} \mathrm{CH}_{2} \cdot \mathrm{SO}_{3} \mathrm{~Pb}+\mathrm{PbHO}$ 47,04 pC.

Der Wassergehalt, welcher gleich 4,0 pC. gefunden wurde, entspricht einem Molecul; berechnet 3,9 pC.

Das neutrale Bleisalz erhielt ich, indern ich zur Lösung der freien Säure Bleioxydhydrat in nur geringer Menge zusetzte, so dafs die Flüssigkeit noch stark sauer reagirte. Beim Erkalten schied sich das Salz in schönen glänzenden Krystallnadeln aus.

Boi dor Wassorbestimmung verloren 1,8873 Grm. Substanz, in einer Temperatur von 100 bis $140^{\circ}, 0,1360$ Wasser, entsprechend $7,2 \mathrm{pC}$.

1,311 Grm. getrocknete Substanz gaben 0,6140 schwefelsaures Blei, entsprechend 31,98 pC. Blei.

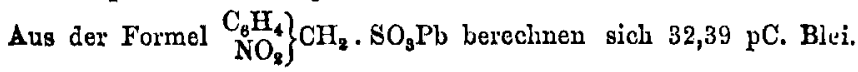

Der Wassergehalt betrug 7,2 pC., entsprechend 11/8 Molecul; berechnet 7,7 pC.

Monoclllorbenzylsulfosäure.

Das bei der Einwirkung von Chlor auf Toluoldampf erhaltene Chlorbenzylchlorid wurde auf dieselbe Weise wie das Chlorbenzyl mil neutralem schwefligsauren Kali behandelt. 
In kurzer Zeit war die Einwirkung der beiden Körper auf einander beendet, worauf die Flüssigkeit filtrirt und etwas verdampft wurde. Beim Erkalten schied sich das Kalisalz in farblosen Krystallnadeln aus.

Durch doppelten Austausch mit Chlorbaryum verwandelte ich dasselbe in monochlorbenzylsulfosauren Baryt, welcher nach mehrmaligem Umkrystallisiren aus heifsem Wasser farblose glänzende Krystalle bildete.

1,1735 Grm. Substanz nahmon, auf $120^{\circ}$ erhitzt, nicht an Gewicht $\mathrm{ab}$; bei einor Temperatur von $180^{\circ}$ verloren sie 0,0147 Wasser, entsprechend $3,1 \mathrm{pC}$.

0,4495 Grm. getrocknete Substanz gaben bei der Behandlung mit concentrirter Schwefelsüure 0,1890 schwefelsauren Baryt.

Die Chlor- und Schwefelbestimmung wurde aus Einer Gewichtsmonge Substanz bestimmt. $\mathrm{Zu}$ diesem Zweck wurden 0,6638 Grm. getrocknete Substanz mit Kalihydrat und Salpeter geschmolzen, das Gemenge in Wasser gelöst und die entstandene Schwefelsture mit salpetersaurem Baryt gefallt. Nach dem Glühen und nochmaligen Behandcln des schwefelsauren Baryts mit Salzsiure ergaben sich 0,5665 schwofclsaurer Baryt.

Aus dem Filtrat des schwefelsauren Baryts wurde das Chlor mit Silberlösung als Chlorsilber gefällt und gewogen. Es betrug $0,3545 \mathrm{Grm}$.

Zum Vergleich stelle ich die gefundenen Resultate mit den aus der Formel $\mathrm{C}_{6} \mathrm{H}_{4} \mathrm{Cl} . \mathrm{CH}_{2} . \mathrm{SO}_{3} \mathrm{Ba}$ berechneten zusammen :

\begin{tabular}{lccc} 
& \multicolumn{2}{c}{ gofunden } & berechnct \\
$\mathrm{C}_{7}$ & 84 & - & - \\
$\mathrm{H}_{6}$ & 6 & - & - \\
$\mathrm{Cl}$ & 35,5 & 13,03 & 12,95 \\
$\mathrm{~S}$ & 32 & 11,72 & 11,68 \\
$\mathrm{Ba}$ & 68,5 & 24,74 & 25,00 \\
$\mathrm{O}_{8}$ & 48 & - & -
\end{tabular}

Aufserdem wurde im Salze 3,1 pC. Wassergehalt gefunden, was $1 / \mathrm{y}$ Molecul entspricht; berechnet 3,1 pC. 
Wie aus obiger Zusammenstellung hervorgeht, ist im Chlorbenzylchlorid nur 1 Atom Chlor durch die Gruppe $\mathrm{SO}_{3} \mathrm{H}$ vertreten worden.

Aus dem Barytsalz stellte ich ein basisches Bleisalz auf dieselbe Weise dar, wie die basischen Bleisalze der Benzylsulfosäure und Nitrobenzylsulfosãure. Es wurde nämlich der Baryt vollständig mit Schwefelsäure ausgefällt und die Lõsung mit Bleioxydhydrat gesättigt. Aus dem Filtrat schied sich beim Erkalten das Salz in Gestalt von kleinen silberglänzenden Krystallschuppen aus.

$0,6355 \mathrm{Grm}$. Substanz, auf $120^{\circ}$ erhitzt, verloren 0,0232 Wasser, entsprechend $3,65 \mathrm{pC}$.

0,6123 Grm. getrocknete Substanz gaben bei der Bestimmung als schwefelsaures Bloi 0,4265 , was 47,59 pC. Blei entspricht. Berechnet aus der Formel $\mathrm{C}_{6} \mathrm{H}_{4} \mathrm{Cl}_{2} \mathrm{CH}_{2} \mathrm{SO}_{3} \mathrm{~Pb}+\mathrm{PbHO}$ 48,19 pC. Blei.

Der gefundene Wassergehalt betrug $3,65 \mathrm{pC}$, der berechnete 4,02 pC., entsprechend einem Molecul.

Durch Zusatz von wenig Bleioxydhydrat zur Lōsung der freien Säure wurde auch ein neutrales Bleisalz in farblosen Krystallschuppen erhalten.

$\mathrm{Da}$ die Einwirkung von neutralem schwefligsaurem Kali auf Benzylchlorid so leicht stattfand, so liefs sich erwarten, auch im Chlorobenzol $\mathrm{C}_{6} \mathrm{H}_{5} . \mathrm{CHCl}_{2}$ die beiden Chloratome der Seitenkelte durch $\mathrm{SO}_{3} \mathrm{H}$ vertreten za können.

Ich stellte daher durch vorsichtige Einwirkung von Phosphorchlorid auf Bittermandelöl Chlorobenzol dar und brachte dasselbe in der Siedehitzc mit neutralem schwefligsaurem Kali zusammen. Die beiden Körper wirkten zwar auf einander ein und es schied sich auch beim Erkalten eine krystallinische Masse aus, die aber gröfstentheils aus der Verbindung von Bittermandelöl mit saurem schwefligsaurem Kali bestand. Es hatte sich daher durch die Einwirkung des Wassers das 
Chlorobenzol zum Theil wieder in Bittermandelöl zurückverwandelt :

$$
\mathrm{C}_{6} \mathrm{H}_{5} \cdot \mathrm{CHCl}_{2}+\mathrm{H}_{2} \mathrm{O}=\mathrm{C}_{6} \mathrm{H}_{5} \cdot \mathrm{CHO}+2 \mathrm{HCl} \text {, }
$$

and die freigewordene Salzsãure verwandelte das neutrale schwefligsaure Kali in saures Salz, das sich mit dem Bittermandelöl verband. Durch Kochen mit verdünnter Schwefelsäure zerstörte ich die Verbindung und erhielt beim Eindampfen und Ausziehen mit Weingeist eine Sulfosăure. Die Ausbeute war jedoch nur gering, so dafs ich die Untersuchung derselben aufgab.

Ueber eine Verbindung von Senföl mit saurem schwefligsaurem Kali;

von Demselben.

Das Senföl, welchem nach Hof mann's Untersuchung die Constitution $\begin{aligned} & \mathrm{C}=\mathrm{I} \\ & \mathrm{N}-\mathrm{C}_{8} \mathrm{H}_{s}\end{aligned}$ zukommt, äufsert zwei freie Affinitäten. Es verbindet sich daher direct mit vielen Körpern, z. B. mit Kaliumsulfhydrat, Ammoniak, mit organischen Basen u. s. w.

Herr Prof. Strecker hat die Beobachtung gemacht, dafs sich dasselbe, ähnlich dem Aldehyd und Bittermandelöl, auch direct mit saurem schwefligsaurem Alkali verbindet, und übertrug mir die nähere Untersuchung dieser Verbindungen.

Da das Natronsalz nicht schön krystallisirte, wandte ich saures schwefligsaures Kali in ziemlich concentrirter Lösung an. Die beiden Körper wurden in äquivalentem Verhältnifs in eine Retorte gebracht und auf dieselbe Weise behandelt, 